\title{
GOLDIE $M$-GROUPS
}

\author{
K. C. CHOWDHURY \\ (Received 15 August 1989; revised 9 May 1990 and 27 September 1990)
}

Communicated by B. J. Gardner

\begin{abstract}
If $(G+)$ is a group and $M$ is a nonempty set of endomorphisms of $G$ operating on the left then $G$ is said to be $M$-Goldie when

(i) $G$ has no infinite independent family of nonzero $M$-subgroups, and

(ii) annihilators in $M$ of subsets of $G$ satisfy the a.c.c. (under set inclusion).

Here we prove some results, analogous to those of a Noetherian module in some special cases, even when the set $M$ of operators has no other algebraic structure than the existence of a zero element or in some cases $M$ is at most a finite dimensional commutative near-ring. Precisely speaking, we prove that the collection of associated operating sets of $G$ is finite and there exists a primary decomposition of 0 of such a Goldie $M$-group, and then if $M$ is a finite dimensional commutative near-ring with unity, for any $x$ belonging to each associated operating set of $G$, a power of it belongs to the annihilator of $G$.

1980 Mathematics subject classification (Amer. Math. Soc.) (1985 Revision): 16 A 34, 16 A 76, 20 B 99.
\end{abstract}

\section{Introduction}

In this paper we introduce the notion of a Goldie operator group and establish some interesting properties of such a system.

If $(G+)$ is a group and $M$ is a nonempty set of endomorphisms of $G$ operating on the left then $G$ is said to be $M$-Goldie when

(i) $G$ has no infinite independent family of nonzero $M$-subgroups, and

(ii) annihilators of subsets of $G$ in $M$ satisfy the ascending chain condition (under set inclusion).

(C) 1991 Australian Mathematical Society $0263-6115 / 91 \$ A 2.00+0.00$ 
A Goldie ring is clearly a Goldie $M$-group. Every finite dimensional left module over a left Noetherian ring is a Goldie $M$-group. An Artinian left module over a left Artinian ring is a Goldie $M$-group.

It can easily be seen that a direct sum of two Goldie $M$-groups is again a Goldie $M$-group. An $M$-subgroup of a Goldie $M$-group is a Goldie $M$ group. But the homomorphic image of a Goldie $M$-group need not be a Goldie $M$-group. For in the case of a Goldie ring, a homomorphic image of a Goldie ring need not be a Goldie ring [2]. A Goldie $M$-group is called fully Goldie if every homomorphic image of it is a Goldie $M$-group.

An $M$-subgroup $H$ of $G$ is called an essential $M$-subgroup of $G$ if for each nonzero $M$-subgroup $K$ of $G, H \cap K \neq 0$. We denote this by $H \leq_{e} G$. Clearly $G \leq_{e} G$ and $0 \leq_{e} G$ if and only if $0=G$. Moreover if $H, K$ are $M$-subgroups of $G, H \subseteq K \subseteq G$, then $H \leq_{e} G$ if and only if $H \leq_{e} K \leq_{e} G$.

If an $M$-subgroup $H$ of $G$ has no proper essential extension inside $G$ (that is, if $H$ and $K$ are $M$-subgroups of $G$ then $H \leq_{e} K<G$ implies $H=K$ ) then $H$ is called a closed $M$-subgroup of $G$ and we write $H \leq_{c} G$. Thus 0 and $G$ are always closed $M$-subgroups of $G$.

An ordered family $\left\{G_{1}, G_{2}, \ldots, G_{n}\right\}$ of $M$-subgroups of $G$ is called an independent family if $\left(G_{1}+\cdots+\widehat{G}_{t}+\cdots+G_{n}\right) \cap G_{t}=0$, for $1 \leq t \leq n$. (The symbol denotes omission of $G_{t}$.)

An $M$-group $G$ is called finite dimensional provided $G$ has no infinite direct sum of nonzero normal $M$-subgroups. To prove $G$ is finite dimensional, it suffices to show that $G$ has no infinite independent sequence of nonzero normal $M$-subgroups.

The annihilator $A(S)$ of a subset $S$ of $G$ is defined as

$$
A(S)=\{m \in M \mid m s=0 \text { for all } s \in S\} .
$$

In our discussion, $M$ will always contain a zero element 0 such that $0 g=0$ for all $g \in G$. Thus $A(S) \neq \varnothing$ for all $S$. A nonzero $M$-subgroup $H$ of $G$ is called a prime $M$-subgroup of $G$ if for every nonzero $M$-subgroup $K$ of $H, A(K)=A(H)$. If, for each $M$-subgroup $H$ of the $M$-group $G$, $A(G)=A(H)$, then $G$ is called a prime $M$-group.

The collection

$$
\mathscr{A}(G)=\{P \subseteq M \mid P=A(H) \text { for some prime } M \text {-subgroup } H \text { of } G\}
$$

is the family of associated operating subsets of $G$. An $M$-group $G$ is $M$ primary if $\mathscr{A}(G)$ is a singleton.

Let $G$ be a Goldie $M$-group with closed normal $M$-subgroups $G_{1}, \ldots, G_{t}$ such that

(1) $G_{1} \cap \cdots \cap G_{t}=0$ and $G_{1} \cap \cdots \cap \widehat{G}_{i} \cap \cdots \cap G_{t} \neq 0$, for $i=1, \ldots, t$ and

(2) each quotient $M$-group $G / G_{i}$ is an $M$-primary group with $\mathscr{A}\left(G / G_{i}\right)$ $\neq \mathscr{A}\left(G / G_{j}\right)$ for $i \neq j$. 
Then $G_{1} \cap \cdots \cap G_{t}$ is called an $M$-primary decomposition of 0 of $G$.

In a unique factorisation domain one can express a non-unit as a finite product $p_{1}^{\alpha_{1}} \cdots p_{n}^{\alpha_{n}}$ of positive powers of distinct primes. This result can be expressed in terms of ideals as $(a)=\left(p_{1}^{\alpha_{1}}\right) \cap \cdots \cap\left(p_{t}^{\alpha_{t}}\right)$.

A similar decomposition of ideals of a commutative Noetherian ring is known. We extend some portions of this theory to Goldie $M$-groups.

Here we prove that if $G$ is a fully Goldie $M$-group and if $\mathscr{A}(G)=X \cup Y$, $X \cap Y=\varnothing$, then in some cases there exists a closed normal $M$-subgroup $G^{\prime}$ of $G$ such that $\mathscr{A}(G)=\mathscr{A}\left(G^{\prime}\right) \cup \mathscr{A}\left(G / G^{\prime}\right)$ where $\mathscr{A}\left(G^{\prime}\right)=X$ and $\mathscr{A}\left(G / G^{\prime}\right)=Y$. Another interesting result is that in some special cases $\mathscr{A}(G)$ is a finite collection. Moreover the very interesting and important result we prove here is the existence of an $M$-primary decomposition of 0 of such a Goldie $M$-group. If $G_{1} \cap \cdots \cap G_{t}$ is such a decomposition of 0 then $\mathscr{A}(G)=\mathscr{A}\left(G / G_{1}\right) \cup \cdots \cup \mathscr{A}\left(G / G_{t}\right)$. Next, if $G$ is a Goldie $M$-group where $M$ is a right near-ring having no infinite direct sum of ideals and is such that $Z_{1}(G)=0$ (where $Z_{1}(G)=\{g \in G \mid I g=0$ for some essential $M$-subgroup $I$ of $G\}$ ) then the annihilators of subsets of $G$ in $M$ satisfy the d.c.c. and if $M$ is a commutative near-ring then for any $x \in \bigcap_{P \in \mathscr{A}(G)} P$ there exists a $t \in \mathbb{Z}^{+}$such that $x^{t} \in A(G)$.

\section{Preliminaries}

Following are some preliminary lemmas for use in the proofs of the main results. First we prove the following important lemma which will play a key role in our theory.

LEMMA 2.1. If an $M$-group $G$ has no infinite independent family of $M$ subgroups then it satisfies the a.c.c. on closed normal $M$-subgroups.

Proof. Suppose $G$ does not satisfy the a.c.c. on closed normal $M$-subgroups. Then $G$ has a chain $G_{1}<G_{2}<\cdots$ of closed normal $M$-subgroups of $G$. Since $G_{n}<{ }_{c} G$, we have $G_{n} \gtrless_{e} G_{n+1}$. Therefore $G_{n+1}$ must have a nonzero $M$-subgroup $C_{n}$ such that $G_{n} \cap C_{n}=0$. And this is true for each $n$. We claim for any $t \in \mathbb{Z}^{+}, i \leq t$, that

$$
\left(C_{1}+\cdots+\widehat{C}_{i}+\cdots+C_{t}\right) \cap C_{i}=0 .
$$

Here

$$
\begin{aligned}
& \left(C_{1}+\cdots+\widehat{C}_{i}+\cdots+C_{t}\right) \cap C_{i} \\
& \quad \subseteq\left(G_{2}+\cdots+G_{i}+C_{i+1}+\cdots+C_{t}\right) \cap C_{i} .
\end{aligned}
$$


Now if $c_{i}=g_{2}+\cdots+g_{i}+c_{i+1}+\cdots+c_{t}$ (where $g_{k} \in G_{k}, k=2, \ldots, i$, and $\left.c_{l} \in C_{l}, l=i, i+1, \ldots, t\right)$ is an element of $\left(G_{2}+\cdots+G_{i}+C_{i+1}+\cdots+C_{t}\right) \cap C_{i}$ then $-c_{i}+g_{2}+\cdots+g_{i}+c_{i+1}+\cdots+c_{t}=0$ implies $g_{2}^{\prime}+\cdots+g_{i}^{\prime}-c_{i}+c_{i+1}+\cdots+$ $c_{t-1}=-c_{t}$ (since $G_{2}, \ldots, G_{i}$ are normal subgroups of $G$ ), where $g_{k}^{\prime} \in G_{k}$, $k=2, \ldots, i$. Thus

$$
c_{t} \in\left(G_{2}+\cdots+G_{i}+G_{i+1}+\cdots+G_{t}\right) \cap C_{t} \subseteq G_{t} \cap C_{t}=0 .
$$

Similarly $c_{t-1}, \ldots, c_{i}$ are all zeros.

So $\left(G_{2}+\cdots+G_{i}+C_{i+1}+\cdots+C_{t}\right) \cap C_{i}=0$ and therefore

$$
\left(C_{1}+\cdots+\widehat{C}_{i}+\cdots+C_{t}\right) \cap C_{i}=0 .
$$

Hence $\left\{C_{1}, C_{2}, \ldots\right\}$ is an independent family of nonzero $M$-subgroups of $G$. Since $G$ has no infinite independent family of $M$-subgroups we can not have a strictly ascending infinite sequence of closed normal $M$-subgroups of $G$. Thus $G$ satisfies the a.c.c. on closed normal $M$-subgroups.

LEMMA 2.2. Let $G$ be an $M$-group satisfying the a.c.c. for annihilators of subsets of $G$ in $M$. Then $A(G) \neq \varnothing$ if and only if $G=0$.

Proof. Suppose $G=0$. Then $G$ has no prime $M$-subgroup. Hence $\mathscr{A}(G)=\varnothing$. Again if $G \neq 0$ consider $\mathscr{H}=\left\{A\left(G^{*}\right) \mid G^{*}\right.$ is an $M$-subgroup of $G\}$. Since $G$ is Goldie, $\mathscr{H}$ has a maximal element, say $A(N)$. Now let $N^{\prime}(\neq 0)$ be an $M$-subgroup of $G$ such that $N^{\prime} \subseteq N$. Then $A\left(N^{\prime}\right) \supseteq A(N)$. So by maximality of $A(N)$ it follows that $A(N)=A\left(N^{\prime}\right)$. Thus $N$ is a prime $M$-subgroup of $G$. Therefore $A(N) \in \mathscr{A}(G)$, that is, $\mathscr{A}(G) \neq \varnothing$.

LEMMA 2.3. Let $G$ be an $M$-group as above with an exact sequence

$$
0 \rightarrow G^{\prime} \stackrel{g}{\rightarrow} G \stackrel{f}{\rightarrow} G^{\prime \prime} \rightarrow 0 .
$$

Then $\mathscr{A}\left(G^{\prime}\right) \subseteq \mathscr{A}(G) \subseteq \mathscr{A}\left(G^{\prime}\right) \cup \mathscr{A}\left(G^{\prime \prime}\right)$.

Proof. If $G=0$ then $G^{\prime}=0$ and $G^{\prime \prime}=0$ and thus the result is true in this case. Assume $G \neq 0$. Since $g$ is injective, $G^{\prime}$ is an $M$-subgroup of $G$. Therefore $\mathscr{A}\left(G^{\prime}\right) \subseteq \mathscr{A}(G)$. Let $A(N) \in \mathscr{A}(G)$ for some prime $M$-subgroup $N$ of $G$. If $N \cap G^{\prime} \neq 0$, then $A\left(N \cap G^{\prime}\right)=A(N)$ since $N$ is a prime $M$-subgroup of $G$ and $N \cap G^{\prime}$, being an $M$-subgroup of the prime $M$-subgroup $N$, is also prime. Therefore $A\left(N \cap G^{\prime}\right) \in \mathscr{A}\left(G^{\prime}\right)$. Thus $A(N) \in \mathscr{A}\left(G^{\prime}\right)$. Now suppose $N \cap G^{\prime}=0$ and $h$ is the restriction of $f$ to $N$. Then $h$ is injective, so $h(N) \cong N \subseteq G^{\prime \prime}$. Thus $A(N) \in \mathscr{A}\left(G^{\prime \prime}\right)$. Hence $\mathscr{A}(G) \subseteq \mathscr{A}\left(G^{\prime}\right) \cup \mathscr{A}\left(G^{\prime \prime}\right)$. 
LEMMA 2.4. Let $N$ be a normal $M$-subgroup of an $M$-group $G$ such that $A$ is a closed $M$-subgroup of $G$ with $N \leq A \leq_{c} G$. Then $A / N \leq_{c} G / N$.

Proof. If not, let $A / N \varsubsetneqq L / N \leq G / N$. Then $N \leq A \lessgtr L \leq G$ and there is an $M$-epimorphism $f: L \rightarrow L / N$.

Here $f^{-1}(A / N)=A$. Since $A / N<_{e} L / N$, it follows that $A<_{e} L$ and this is not possible for $A \leq_{c} G$. Hence $A / N \leq_{c} G / N$. The following two lemmas are easy to prove.

Lemma 2.5. If $G_{1}$ and $G_{2}$ are two Goldie $M$-groups then

$$
\mathscr{A}\left(G_{1} \oplus G_{2}\right)=\mathscr{A}\left(G_{1}\right) \cup \mathscr{A}\left(G_{2}\right) \text {. }
$$

LEMMA 2.6. If $G$ is a Goldie $M$-group and $P, Q, N$ are $M$-subgroups of $G, N \triangleleft G$ such that $N \leq P, Q$ then $\mathscr{A}(P \cap Q / N)=\mathscr{A}(P / N \cap Q / N) \subseteq$ $\mathscr{A}(P / N) \cap \mathscr{A}(Q / N)$.

Let $H$ and $K$ be two $M$-subgroups of an $M$-group $G$ such that $H \leq$ $K \leq G$. Then $H$ is $M$-essential in $K$ if for any $M$-subgroup $L(\subseteq K)$, $H \cap L \neq 0$.

We now consider the set $M$ of operators as a right near-ring with 1 such that $1 g=g,\left(m_{1}+m_{2}\right) g=m_{1} g+m_{2} g,\left(m_{1} m_{2}\right) g=m_{1}\left(m_{2} g\right)$ for $g \in G$, $m_{1}, m_{2} \in M$ (in other words, $G$ is a left near module over the right near-ring $M)$.

LEMMA 2.7. Let $N$ and $H$ be $M$-subgroups of an $M$-group $G$ such that $H$ is $M$-essential in $N$. If $a \in N, a \neq 0$, then there is an essential left $M$-subgroup $L$ of $M$ such that $L a \neq 0, L a \subseteq H$.

Proof. Let $L=\{m \in M \mid m a \in H\}$. Then $L$ is left $M$-subgroup of $M$ and $M a \subseteq N$ (since $N$ is an $M$-subgroup of $G$ and $a \in N$ ). Also $M a \neq 0$ (for $1 \in M$ Implies $a \in M a$ ). Since $H$ is $M$ essential in $N$, we get $M a \cap H \neq 0$. Let $h=m a(\neq 0) \in H$. So $L a \neq 0$. We now show that $L$ is an essential left $M$-subgroup of $M$. Let $I(\neq 0)$ be a left $M$-subgroup of $M$. We claim that $I \cap L \neq 0$. Suppose $I a=0$. Then $I a \subseteq H$. So $I \subseteq L$. Hence $I \cap L \neq 0$. And if $I a \neq 0$ then $I a$ is an $M$-subgroup of $G$ and $I a \subseteq N$. Since $H$ is $M$-essential in $N, \operatorname{Ia\cap H} H \neq 0$. Hence for some $x(\neq 0) \in I, x a \in H$. Thus $x \in L$. Therefore $I \cap L \neq 0$ which implies that $L$ is an essential left $M$-subgroup of $M$.

We define

$$
Z_{1}(G)=\{x \in G \mid A x=0 \text { for some essential left } M \text {-subgroup } A \text { of } M\}
$$


and for any $S(\subseteq M)$,

$$
\mathrm{r}_{G}(S)=\{g \in G \mid s g=0 \text { for all } s \in S\} .
$$

Lemma 2.8. Let $P, Q$ be annihilators of subsets of $G$ in $M$ such that $P \subseteq Q$ and $P$ is $M$-essential in $Q$. If $Z_{1}(G)=0$ then $P=Q$.

Proof. Let $q \in Q, q \neq 0$. Since $P \subseteq Q$ and $P$ is $M$-essential in $Q$ there exists an essential left $M$-subgroup $L$ of $M$ such that $L q \in P$. $L q \neq 0$ (Lemma 2.7). thus $L q \mathrm{r}_{G}(P)=0$. So $q \mathrm{r}_{G}(P)=0$ implies $q \in A$ $\left(\mathrm{r}_{G}(P)\right)=P($ since $P$ is an annihilator of a subset of $G$ in $M)$. Hence $P=Q$.

We see that the $\mathbb{Z}$ groups $\mathbb{Z}_{3}, \mathbb{Z}_{6}, \mathbb{Z}_{15}$, etc. are such $\mathbb{Z}$ Goldie groups that their proper quotients are all prime $\mathbb{Z}$-groups. And in a prime $M$-group all of its $M$-subgroups are prime and at least the $M$-group itself is a prime closed extension of each of its prime $M$-subgroups. Again $G=\mathbb{Z}_{30}$ is such a Goldie $\mathbb{Z}$-group that its $\mathbb{Z}$-subgroups are

$$
\begin{aligned}
A_{1}=\{0,2,4, \ldots, 28\}, & A_{2}=\{0,3,5, \ldots, 27\}, \\
A_{3}=\{0,5,10, \ldots, 25\}, & A_{4}=\{0,6,12, \ldots, 24\}, \\
A_{5}=\{0,10,20\}, & \text { and } A_{6}=\{0,15\}
\end{aligned}
$$

of which $A_{4}<A_{2}<{ }_{c} G, A_{5}<A_{3}<{ }_{c} G$ and $A_{6}<A_{3}<{ }_{c} G$. So by Lemma 2.4, $A_{2} / A_{4}<_{c} G / A_{4}, A_{3} / A_{5}<_{c} G / A_{5}$ and $A_{3} / A_{6}<_{c} G / A_{6}$.

Here each of $A_{2} / A_{4}, A_{3} / A_{5}$ and $A_{3} / A_{6}$ is a prime $M$-subgroup. Thus each of a closed extension of itself which is prime. And the remaining quotients $G / A_{1}, G / A_{2}$ and $G / A_{3}$ are all primes.

These are such Goldie $M$-groups that any prime $M$-subgroups $N / G^{\prime}$ of $G / G^{\prime}$ has a prime closed normal extension $T / G^{\prime}$ such that $G^{\prime} \leq N \leq T \leq_{c}$ $G$.

In what follows our Goldie $M$-group $G$ will be of this type.

\section{Main results}

TheOREM 3.1. Let $G$ be a Goldie $M$-group described as above. If the set $\mathscr{A}(G)$ is a union of two disjoint sets $X$ and $Y$, then there exists a normal closed $M$-subgroup $G^{\prime}$ such that $\mathscr{A}\left(G^{\prime}\right)=X$ and $\mathscr{A}\left(G / G^{\prime}\right)=Y$.

Proof. Let $\mathscr{H}=\left\{N \unlhd_{c} G \mid \mathscr{A}(N) \subseteq X\right\}$. As 0 is a closed normal $M$-subgroup of $G$ and $\mathscr{A}(0)=\varnothing$, we have $\mathscr{H} \neq \varnothing$ (since $\varnothing \subseteq X$ ).

Since $G$ is $M$-Goldie, by Lemma $2.1, \mathscr{H}$ has a maximal element, (say) $G^{\prime}$. Also, $X \cup \mathscr{A}\left(G / G^{\prime}\right) \supseteq X \cup Y$ (Lemma 2.3). Since $X \cap Y=\varnothing$, we have 
$Y \subseteq \mathscr{A}\left(G / G^{\prime}\right)$. Suppose $\mathscr{A}\left(G / G^{\prime}\right) \nsubseteq Y$. Then there exists a prime $M$ subgroup $N / G^{\prime}$ of $G / G^{\prime}$ such that $A\left(N / G^{\prime}\right) \in \mathscr{A}\left(G / G^{\prime}\right)$ and $A\left(N / G^{\prime}\right) \notin Y$. Moreover by hypothesis there is a prime closed $M$-normal extension $T / G^{\prime}$ such that $N / G^{\prime} \leq T / G^{\prime} \unlhd_{c} G / G^{\prime}$ and $G^{\prime} \leq N \leq T \unlhd_{c} G$. Thus $T$ is a closed normal $M$-subgroup of $G$. Since $T / G^{\prime}$ is nonzero, $G^{\prime} \subseteq T$ and $A\left(T / G^{\prime}\right)=$ $A\left(N / G^{\prime}\right)$. Since $T / G^{\prime}$ is prime, $\mathscr{A}\left(T / G^{\prime}\right)$ is a singleton set, say $\{P\}$. We write simply $P$. Thus $\mathscr{A}\left(T / G^{\prime}\right)=P$ and $P \notin Y$. Again by Lemma 2.4, $\mathscr{A}(T) \subseteq \mathscr{A}\left(G^{\prime}\right) \cup \mathscr{A}\left(T / G^{\prime}\right)$. Since $\mathscr{A}\left(G^{\prime}\right) \subseteq X$ and $\mathscr{A}\left(T / G^{\prime}\right)=P$, we get $\mathscr{A}(T) \subseteq X \cup P$. Also $T \subseteq G$ and $\mathscr{A}(G)=X \cup Y$ give $\mathscr{A}(T) \subseteq X \cup Y$.

So $P \notin Y$ gives $\mathscr{A}(T) \subseteq X$. Thus $T \in \mathscr{H}$ and this contradicts the maximality of $G$. Therefore $\mathscr{A}\left(G / G^{\prime}\right) \subseteq Y$. Thus $X \cup Y \subseteq \mathscr{A}\left(G^{\prime}\right) \cup Y$ and $X \cap Y=\varnothing$ gives $X \subseteq \mathscr{A}\left(G^{\prime}\right)$.

TheOREM 3.2. Let $G$ be a Goldie $M$-group as above. Then $\mathscr{A}(G)$ is finite.

Proof. We assume the opposite, that is, that $\mathscr{A}(G)=\{P, Q, R, \ldots\}$ is infinite.

If $\mathscr{A}(G)=P \cup Y$ and $P \notin Y$ (we write $P$ for $\{P\}$ ) then by Theorem 3.1 there exists a closed normal $M$-subgroup $G^{\prime}$ of $G$ such that $\mathscr{A}\left(G^{\prime}\right)=P$, $\mathscr{A}\left(G / G^{\prime}\right)=Y$. Thus

$$
\mathscr{A}(G)=\mathscr{A}\left(G^{\prime}\right) \cup \mathscr{A}\left(G / G^{\prime}\right) .
$$

Since $Q \in \mathscr{A}(G)$ we have $Q \in \mathscr{A}\left(G / G^{\prime}\right)$, so for some prime $M$-subgroup $B^{\prime} / G^{\prime}$ of $G / G^{\prime}, A\left(B^{\prime} / G^{\prime}\right)=Q$. Thus $\mathscr{A}\left(B^{\prime} / G^{\prime}\right)=Q$. By hypothesis there is a prime extension $G^{\prime \prime} / G^{\prime}$ such that $B^{\prime} / G^{\prime}<G^{\prime \prime} / G^{\prime} \unlhd_{c} G / G^{\prime}$ and $G^{\prime} \leq$ $B^{\prime} \leq G^{\prime \prime} \unlhd_{c} G$. Hence $A\left(G^{\prime \prime} / G^{\prime}\right)=A\left(B^{\prime} / G^{\prime}\right)=Q$. Therefore $\mathscr{A}\left(G^{\prime \prime} / G^{\prime}\right)=$ $\mathscr{A}\left(B^{\prime} / G^{\prime}\right)=Q$. And by Lemma $2.3, \mathscr{A}\left(G^{\prime \prime}\right) \subseteq \mathscr{A}\left(G^{\prime}\right) \cup \mathscr{A}\left(G^{\prime \prime} / G^{\prime}\right)$. It follows that $\mathscr{A}\left(G^{\prime \prime}\right) \subseteq\{P, Q\}$. Also by Lemma $2.3, \mathscr{A}(G) \subseteq \mathscr{A}\left(G^{\prime \prime}\right) \cup$ $\mathscr{A}\left(G / G^{\prime \prime}\right)$. Therefore $\mathscr{A}(G) \subseteq\{P, Q\} \cup \mathscr{A}\left(G / G^{\prime \prime}\right)$, that is, $R \in\left(G / G^{\prime \prime}\right)$.

In a like manner we get another closed normal $M$-subgroup $G^{\prime \prime \prime}$ of $G$ such that $G^{\prime}<G^{\prime \prime}<G^{\prime \prime \prime}$ and for $S \in \mathscr{A}(G), S \in \mathscr{A}\left(G / G^{\prime \prime \prime}\right)$. Since $\mathscr{A}(G)$ is infinite, we get a strictly ascending infinite sequence of closed normal $M$ subgroups, which contradicts the Goldie character of $G$ because of Lemma 2.1. Hence $\mathscr{A}(G)$ is finite.

THEOREM 3.3. Let the $M$-group $G$ be fully Goldie as above.

(I) There exists an $M$-primary decomposition of 0 in $G$.

(II) If $G_{1} \cap \cdots \cap G_{t}$ is an $M$-primary decomposition of 0 in $G$ then $\mathscr{A}(G)=\mathscr{A}\left(G / G_{1}\right) \cup \cdots \cup \mathscr{A}\left(G / G_{t}\right)$.

Proof. (I) By the above theorem, $\mathscr{A}(G)$ is finite. 
Let $\mathscr{A}(G)=\left\{P_{1}, \ldots, P_{t}\right\}$. Since $\mathscr{A}(G)$ is expressible as a union of two disjoint sets $\left\{P_{1}, \ldots, \widehat{P}_{i}, \ldots, P_{t}\right\}$ and $\left\{P_{i}\right\}$, by Theorem 3.1, we get closed normal $M$-subgroups $G_{1}, \ldots, G_{t}$ of $G$ such that for each $i$,

$$
\mathscr{A}\left(G_{i}\right)=\left\{P_{1}, \ldots, \widehat{P}_{i}, \ldots, P_{t}\right\} \text { and } \mathscr{A}\left(G / G_{i}\right)=\left\{P_{i}\right\} \text {. }
$$

Also, for each $i, G / G_{i}$ is $M$-primary and $\mathscr{A}\left(G / G_{i}\right) \neq \mathscr{A}\left(G / G_{j}\right)$ for $i \neq j$ and $\mathscr{A}\left(G_{1} \cap \cdots \cap G_{i}\right) \subseteq \mathscr{A}\left(G_{1}\right) \cap \cdots \cap \mathscr{A}\left(G_{t}\right)$. Since clearly $\mathscr{A}\left(G_{1}\right) \cap \cdots \cap$ $\mathscr{A}\left(G_{t}\right)=\varnothing$, we then have $\mathscr{A}\left(G_{1} \cap \cdots \cap G_{t}\right)=\varnothing$ and therefore by Lemma 2.2, $G_{1} \cap \cdots \cap G_{t}=0$. If possible let $G_{1} \cap \cdots \cap \widehat{G}_{i} \cap \cdots \cap G_{t}=0$, that is, $\bigcap_{j \neq i} G_{j}=0$, for some $i, 1 \leq i \leq t$. Then we get an $M$-homomorphism.

$$
\alpha: G \rightarrow \bigoplus_{j \neq i} G_{j}, \quad g \mapsto\left(g+G_{1}, \ldots, \widehat{g+G_{i}}, \ldots, g+G_{t}\right) .
$$

We note that $\operatorname{Ker} \alpha=\left\{g \mid g \in \bigcap_{j \neq i} G_{j}=0\right\}=0$. Thus $\alpha$ is an embedding and hence $\mathscr{A}(G) \subseteq \mathscr{A}\left(\bigoplus_{j \neq i} G / G_{j}\right)$. Since $G$ is fully Goldie, each $G / G_{i}$ is Goldie. so it follows from Lemma 2.5 that for each $i, \mathscr{A}(G) \subseteq$ $\bigcup_{j \neq i} \mathscr{A}\left(G / G_{j}\right)$, that is, $\mathscr{A}(G) \subseteq\left\{P_{1}, \ldots, \widehat{P}_{i}, \ldots, P_{t}\right\}$ which is absurd. Hence $\bigcap_{j \neq i} G_{j} \neq 0$.

(II) Next suppose that $\bigcap_{j=1}^{t} G_{j}$ is an $M$-primary decomposition of 0 in $G$. Then the map

$$
\alpha: G \rightarrow \bigoplus_{j=1}^{t} G / G_{j}, \quad g \mapsto\left(g+G_{1}, \ldots, g+G_{t}\right)
$$

is an embedding, which means that $\mathscr{A}(G) \subseteq \mathscr{A}\left(\bigoplus G / G_{j}\right)$ and hence $\mathscr{A}(G) \subseteq$ $\bigcup \mathscr{A}\left(G / G_{j}\right)$. To see the opposite inclusion consider the $M$-homomorphism

$$
\beta: \bigcap_{j \neq i} G_{j} \rightarrow G / G_{i}, \quad g \mapsto g+G_{i} .
$$

Now $\operatorname{Ker} \beta=\left\{g \mid g \in G_{j}\right\}=0$. Thus $\mathscr{A}\left(\bigcap_{j \neq i} G_{j}\right) \subseteq \mathscr{A}\left(G / G_{i}\right)$ and by Lemma $2.2, \mathscr{A}\left(\bigcap_{j \neq i} G_{j}\right) \neq \varnothing$. Since $\mathscr{A}\left(G / G_{i}\right)$ is a singleton, we get $\mathscr{A}\left(\bigcap_{j \neq i} G_{j}\right)=\mathscr{A}\left(G / G_{i}\right)$ for each $i$. Hence

$$
\bigcup_{j=1}^{t} \mathscr{A}\left(G / G_{j}\right)=\bigcup_{i=1}^{t}\left(\mathscr{A}\left(\bigcap_{j \neq i} G_{j}\right)\right.
$$

and since $\mathscr{A}\left(\bigcap_{j \neq i} G_{j}\right) \subseteq \mathscr{A}(G)$ for each $i$, we finally get $\bigcup_{j=1}^{t} \mathscr{A}\left(G / G_{j}\right) \subseteq$ $\mathscr{A}(G)$. Thus $\mathscr{A}(G)=\bigcup_{j=1}^{t} \mathscr{A}\left(G / G_{j}\right)$.

We now give two results on a Goldie $M$-group when the operating set $M$ is a right near-ring with no infinite direct sum of left ideals and $Z_{1}(G)=0$. Theorem 7 of Oswald [5] follows as a corollary to the following result in the case of a regular left Goldie near-ring [3]. 
TheOREM 3.4. Let $G$ be a Goldie $M$-group with $Z_{1}(G)=0$ as above and such that an essential left ideal of $M$ is essential as a left $M$-subgroup also. Then the annihilators of subsets of $G$ in $M$ satisfy the d.c.c.

Proof. Let $B=A(Y), C=A(X), X, Y \subseteq G$. Then if $X \subseteq Y$ we have $B \subseteq C$. Suppose $B \subset C$. Then by Lemma 2.8 , there exists a left $M$ subgroup $D$ of $M$ such that $D \subseteq C, B \cap D=0$. Thus if in the descending chain $A\left(S_{1}\right) \supseteq A\left(S_{2}\right) \supseteq \cdots$ we have $A\left(S_{k}\right) \supseteq A\left(S_{k+1}\right)$, then there exists left $M$-subgroups $P_{k}$ such that $P_{k} \subseteq A\left(S_{k}\right)$ and $A\left(S_{k+1}\right) \cap P_{k}=0$. Again we choose a left ideal $X_{k}$ such that $A\left(S_{k+1}\right) \cap X_{k}=0$ and $X_{k}$ is maximal for this.

Being the left annihilator of $S_{k+1}$ in $M, A\left(S_{k+1}\right)$ is a left ideal of $M$. So $A\left(S_{k+1}\right)+X_{k}$ is a left ideal of $M$. So it is essential as a left $M$-subgroup. Therefore $P_{k} \cap\left(A\left(S_{k+1}\right)+X_{k}\right) \neq 0$ (we write $A_{k}$ for $A\left(S_{k}\right)$ ). Now let $(0 \neq) \quad b_{k} \quad\left(\in P_{k}\right)=a_{k+1}+x_{k}, a_{k+1} \in A_{k+1}, x_{k} \in X_{k}$. This implies $x_{k}=-a_{k+1}+b_{k} \in A_{k+1}+P_{k} \subseteq A_{k}+P_{k} \subseteq A_{k} \cap X_{k} \quad\left(=C_{k}\right.$, say). Now if $x_{k}$ were 0 , we would have $a_{k+1}=b_{k} \in P_{k} \cap A_{k+1}=0$. So $x_{k} \neq 0$. Therefore we get a nonzero left ideal $C_{k}$ and $C_{k} \cap A_{k+1}=0$. An infinite descending chain of left annihilators of subsets of $G$ in $M$ gives an infinite direct sum of left ideals of $M$. Since $M$ has no infinite direct sum of left ideals, the descending chain $A_{1} \supseteq A_{2} \supseteq \cdots$ is a finite one. Now we prove our last result of this paper, in the case of a finite dimensional commutative near-ring with 1 .

THEOREM 3.5. Let $G$ be a Goldie $M$-group where $M$ is a commutative near-ring with 1 having no infinite direct sum of ideals and is such that $Z_{1}(G)=0$. Then for any $x \in \bigcap_{p \in \mathscr{A}(G)} P$, there exists $t \in \mathbb{Z}^{+}$such that $x^{t} \in A(G)$.

Proof. Let $x \in \bigcap_{p \in \mathscr{A}(G)} P$. Then for every positive integer $i$, we get $M$-homomorphisms $\varphi_{i}: G \rightarrow G, g \mapsto x^{i} g, i=1,2, \ldots$ Clearly $\operatorname{Ker} \varphi_{i} \subseteq$ $\operatorname{Ker} \varphi_{i+1}$. In other words $\mathrm{r}_{G}\left(x^{i}\right) \subseteq \mathrm{r}_{G}\left(x^{i+1}\right)$ which gives

$$
A\left(\mathrm{r}_{G}\left(x^{i}\right)\right) \supseteq A\left(\mathrm{r}_{G}\left(x^{i+1}\right)\right) \text {. }
$$

By Theorem 3.4, we get $A\left(\mathrm{r}_{G}\left(x^{r}\right)\right)=A\left(\mathrm{r}_{G}\left(x^{t+1}\right)\right)$ for some $t \in \mathbb{Z}^{+}$. Then $\mathrm{r}_{G}\left(A\left(\mathrm{r}_{G}\left(x^{t}\right)\right)\right)=\mathrm{r}_{G}\left(A\left(\mathrm{r}_{G}\left(x^{t+1}\right)\right)\right)$, that is, $\mathrm{r}_{G}\left(x^{t}\right)=\mathrm{r}_{G}\left(x^{t+1}\right)$ on $\operatorname{Ker} \varphi_{t}=$ $\operatorname{Ker} \varphi_{t+1}$. Now we consider the $M$-homomorphism

$$
f: x^{t} G \rightarrow x^{t} G, \quad x^{t} g \mapsto x^{t+1} g .
$$

If $x^{t+1} g=x^{t+1} g^{\prime}$ then $x^{t+1}\left(g-g^{\prime}\right)=0$ so $g-g^{\prime} \in \operatorname{Ker} \varphi_{t+1}=\operatorname{Ker} \varphi_{t}$ and thus $x^{t} g=x^{t} g^{\prime}$. Hence $f$ is injective. Now $x^{t} G \leq G$ so $\mathscr{A}\left(x^{t} G\right) \subseteq \mathscr{A}(G)$. 
If $x^{t} G \neq 0$ then $\mathscr{A}\left(x^{t} G\right) \neq \varnothing$. Then there exists a nonzero $M$-subgroup $G^{\prime}$ of $x^{t} G$ such that $A\left(G^{\prime}\right) \in \mathscr{A}\left(x^{t} G\right)$. Since $x \in P$ for each $P \in \mathscr{A}(G)$, we get $x \in P$ for each $P \in \mathscr{A}\left(x^{t} G\right)$. So $x \in A\left(G^{\prime}\right)$. And this gives that $x G^{\prime}=0$, that is, $f\left(G^{\prime}\right)=0$. Since $f$ is injective, it follows that $G^{\prime}=0$, a contradiction. Hence $x^{t} G=0$, that is, $x^{t} \in A(G)$.

\section{Acknowledgement}

Thanks are due to Professor B. P. Chetiya, Gauhati University, Guwahati, India, for the valuable discussion the author had with him during the preparation of the paper.

The author is thankful to the referee for his valuable remarks and suggestion.

\section{References}

[1] J. Barshay, Topics in ring theory, W. A. Benjamin, New York, 1969.

[2] A. W. Chatters and C. R. Hazarnavis, Rings with chain conditions, Pitman Advanced Publishing Program, Boston, 1980.

[3] K. C. Chowdhury and A. Masum, 'A note on regular left Goldie near-rings', Nat. Acad. Sci. Lett. (India) 12 (1989).

[4] A. V. Jategaonkar, Left principal ideal rings, Springer-Verlag Lecture Notes No. 123, 1970.

[5] A. Oswald, 'Near rings with chain conditions on right annihilators', Proc. Edinburgh Math. Soc. 23 (1980), 123-127.

Gauhati University

Guwahati 781014

Assam

India 